\title{
Coronary Lesions Treated
}

National Cancer Institute

\section{Source}

National Cancer Institute. Coronary Lesions Treated. NCI Thesaurus. Code C80475.

The coronary lesions treated during a PCI (Percutaneous Coronary Intervention)

procedure. 